\title{
La espiritualidad de la educación integral como factor de bienestar bioético y sostenible
}

\section{(The spirituality of integral training as a bioethical and sustainable welfare factor)}

\section{JUAN MANUEL PINEDA-ALBALADEJO ${ }^{1}$, JORGE LÓPEZ PUGA ${ }^{2}$, FRANCISCO JOSÉ MOYA-FAZ ${ }^{3}$}

${ }^{1}$ Psy, MSc., Doctorando. Programa Doctorado de Ciencias de la Salud. EIDUCAM. ORCID: https://orcid.org/0000-0001-6476-8295

${ }^{2}$ Psy, PhD, Profesor, Departamento de Ciencias de la Salud. UCAM.

ORCID: https://orcid.org/0000-0003-0693-0092

${ }^{3}$ Psy, PhD, Profesor, Catedrático Psicogeriatría. UCAM.

ORCID: https://orcid.org/0000-0002-5832-4900

Universidad Católica de Murcia (UCAM).

Campus Los Jerónimos, 135, Guadalupe

30107 Murcia, España

juanmanuelpinedaalbaladejo@gmail.com

Resumen. El evidente alejamiento entre los postulados religiosos y la actividad secular nos ha llevado al desorden espiritual y al olvido de las necesidades y aspiraciones auténticas del ser humano. La mentalidad y la praxis actuales, hacia lo que se considera meramente útil o verificable, nos ha llevado a esta antinomia de extremos polarizados, 
ya sea hacia un antropocentrismo que sobrevive en una felicidad paradójica, desproporcionada en la búsqueda de lo estrictamente tangible, o hacia un discurso económico o ecológico que olvida la idea ontológica, y el auténtico valor y significado del ser humano. Desde un análisis positivo y normativo, este estudio pretende enfatizar la importancia de la llamada Educación Integral, impartida por algunos centros universitarios, como promotora del bienestar psicológico y espiritual, así como de las actitudes de emprendimiento sostenible, de forma ética y responsable.

Palabras clave: educación integral; ética; emprendimiento; sostenibilidad; universitarios.

Abstract. The evident remoteness between the religious postulates and the secular activity has led us to spiritual disorder and the oblivion of the authentic needs and aspirations of the human being. The current mentality and practice, towards what is considered merely useful or verifiable, has led us to this antinomy of polarized extremes, either towards an anthropocentrism that survives in a paradoxical happiness, disproportionate in the search for the strictly tangible, or towards an economic or ecological discourse that forgets the ontological idea, and the authentic value and meaning of the human being. From a positive and normative analysis, this study aims to emphasize the importance of the so-called Integral Training, taught by some university centers, as a promoter of the psychological and spiritual well-being, as well as the attitudes of sustainable entrepreneurship, in an ethical way and responsible.

Keywords: entrepreneurship; ethics; integral training; sustainability; university.

\section{Introducción}

Que las sinergias del modelo de desarrollo actual den como resultado la denominada "Crisis de la Civilización”, no es nada nuevo. El desequilibrio imperante causado por el ser humano ante sus demandas y necesidades, acompañado de sus conductas irresponsables hacia el medio natural persona-sociedad-ecosistema que las satisface, muestran el alejamiento del claro sentido de la vida y de la dignidad humana, por la falta de ética, justicia, solidaridad, y responsabilidad moral en todos los ámbitos de actuación. Y es que, aún siendo el propio ser humano garante de proponer soluciones y corregir los sesgos causantes de tal inestabilidad, se enfrenta ante un desencuentro consensual de compleja solución. La desconsideración de los términos "bioética", "emprendimiento", y "sostenibilidad", entre otros, de forma conjunta, como constructos de urgente pertinencia global, es una clara muestra de ello. A pesar de la amenaza de una re-humanización ensimismada por la técnica, vaga la razón sin fe, ilusa de su propia omni- 
potencia, creyendo por su inherente autonomía y libertad, capaz de dar respuestas amables a todo (Congregación para la Doctrina de la Fe 2008, 858-887). Intentamos corregir no desde las propias bases de nuestra cultura, sino por cualquier técnica ad hoc que, adornada de progreso o evolución, favorezca las expectativas del impredecible e insaciable homo consumericus (Lipovetsky 2007; Saad 2007), atado a la columna de sus vicios considerados como problemas o necesidades. Hemos llegado a considerar como plausibles aquellas intenciones y conductas que conllevan un acto inmoral, justificando un fin que se presume mayor. Se nos olvida incluso aquello de que, aún siendo moralmente lícitos ciertos actos, no siempre es lo mejor, llegando a la manipulación del axioma de que "el fin justifica los medios", en contra de las teorías deontológicas de la ética.

¿A dónde nos han llevado los sincretismos ideológicos? A dejar de buscar en la actividad interior del ser humano, en el ethos de la persona, la esencia misma de la vida ética; allí donde la perfección de esta es un valor moral que conlleva la marca de la causalidad. No bastan inferencias fenomenológicas, manifestadas en la constante peculiaridad de la experiencia de vida personal para determinar el principio objetivo por el que un acto es éticamente bueno o malo, sino que es necesario reconocer que la ética también está sometida al orden sobrenatural. Y toda interpretación científica vinculada al orden sobrenatural, debe ser interpretada por un sistema teológico (Wojtyla 1982).

En nuestro estudio reflexionaremos sobre la tendencia y estado de las iniciativas sostenibles en la universidad, la necesidad de priorizar a la persona en su integridad, y el valor de la dimensión espiritual cristiana a través de la formación en Ética fundamental y aplicada, Teología, Doctrina Social de la Iglesia, Bioética, y Humanidades, denominada: Educación Integral.

\section{La Educación Integral y la Universidad Sostenible}

La idea de "dar luz" y potenciar la razón son formas indirectas de influencia y de facultad del pensamiento de la educación. Desde la Sección de la Educación para el Desarrollo Sostenible, organismo de la UNESCO, se expresa claramente que, dicha educación: "por sí sola no será suficiente para lograr 
un futuro más sostenible, sin embargo, sin la educación y el aprendizaje para el Desarrollo Sostenible, no podremos lograr esta meta” (UNESCO 2007). Nos preguntamos qué está ocurriendo en los estadios académicos superiores, donde urge el diálogo entre la teología y el conocimiento científico, ante la verdad única e irrefutable (Martínez 2019, 202). ¿Resulta evidente la priorización de la persona en su integridad? ¿Qué puede aportar la universidad?

Ante el reto de alcanzar una educación considerada de calidad y excelencia, las instituciones universitarias tienden a una renovación, que atienda a los desafíos de la sociedad actual, donde el emprendimiento sostenible y las actitudes que median en su intención, pasan a formar parte de la identidad de la propia institución. En un ambicioso estudio mundial realizado por Lozano et al. (2015), se nos mostraba que un $85 \%$ de las universidades, estaban implicadas en materias de sensibilización medioambiental, donde la participación de estudiantes universitarios españoles en jornadas o actividades sostenibles o socio-ambientales, eran cercanas al 90\%. A pesar de estas esperanzadoras cifras que no nos garantizan la evitación de un greenwashing, como estrategia de encubrimiento de otras conductas nocivas, la diversidad de propuestas existentes sin un marco de referencia común, las formas inmediatistas de entender la economía, el comercio y la producción, así como el desorden espiritual en el que se estructura nuestra inteligencia, se ajustan más a un paradigma verde-tecnocrático, en detrimento del auténtico humanismo y de la ética aplicada a la vida humana.

Olvidamos al cristianismo como lugar de encuentro, donde el ser humano está íntimamente vinculado e integrado con el valor de la naturaleza. Donde concurre causalidad con consecuencia moral, con uno mismo y con los demás (Puig 2019, 73-96). Ello da lugar a encontramos con competentes profesionales, que desde su ética substancialista o causalista están más preocupados por la flora o la fauna que de la propia dignidad humana. O tal como expresaba el profesor emérito Raymundo Llanio, los buenos médicos, que no médicos buenos, que no tienen reparo alguno en el ejercicio abortista o eutanásico, al margen de una bioética integral como ciencia de la vida; políticos o economistas sobresalientes, inmersos en la corrupción que causan un daño social de proporciones degradantes. Ante esta pandemia, ¿a qué 
pueden aspirar los estadios formativos superiores, en una sociedad víctima de una falsa filosofía deshumanizada, enferma en su conciencia e inteligencia? Desde una crítica racional, el vacío moral, la trascendente sinrazón producida por las dimensiones funcionalista y economicista actuales, y la crisis ecológica como fruto de una crisis antropológica y espiritual, nos muestra la urgente necesidad de retomar la promoción del factor humano, en búsqueda de su vocación y sentido necesario, a fin de que trascienda al contexto global de forma ordenada, ecuánime y justa.

En palabras de Benedicto XVI, "el primer capital que se ha de salvaguardar y valorar es el hombre, la persona en su integridad” (Benedicto XVI 2009, 25). Por tanto, la educación adquiere un papel esencial desde el núcleo familiar, hasta las etapas de formación superior como enriquecedores del capital humano, sea la fe, sea la razón. Y es aquí, donde la Educación Integral tiene suficiente autoridad para el tratado de aquellos factores ontológicos que operan sobre las actitudes y la conducta, en los que la moral y la ética son sus pilares, y sobre los que se cimenta el potencial sujeto proactivo y sostenible. Todo ello nos conduce a la reflexión de que, las instituciones universitarias no debieran permanecer ajenas, sumidas en pragmatismos, o incapaces de justificar una fe real de una inspiración espiritual. Estas se ha de convertir en una matriz facilitadora del apoyo incondicional a la vida, a la comunidad universal, y de las demandas latentes de la naturaleza, como obligación moral que asume que no todo es verificable desde la experiencia sensible, ni todo debe ser una idea 'fenomelizada' del ser humano. Antes bien, procede la constitución de la verdad, a través de la demostración razonable y la veracidad experimental, más allá de la dominación pragmática. Una muestra de ello, se recoge en las palabras del neotomista contemporáneo Jacques Maritain $(1943,15)$ :

Si nosotros y la juventud que será educada para las futuras democracias, consideramos que todo lo que no es calculable o materialmente ejecutable no es sino un mito; entonces resulta muy vano luchar contra toda forma de totalitarismo, ya que el prefacio al totalitarismo es el desconocimiento de la dignidad espiritual del hombre, y el postulado de que la vida y la moralidad humanas están reguladas por valores puramente materiales o biológicos. 
De igual modo, en nuestra propia crisis reconocemos la importancia de una revisión del modelo curricular universitario, que implemente a sus políticas de excelencia el desarrollo sostenible más allá de la educación ambiental, desde un marco de referencia claro y común, con la debida evaluación y cumplimiento de las funciones educativas y sociales, que son inherentes de dichas instituciones. La universidad, en ningún caso, puede pretender transformar a los futuros profesionales, la peculiar e irreemplazable vocación y sentido de su ser humano, obnubilando y saturando a los sujetos con teorías abstractas, o prácticas conducentes a un mero desarrollo técnico, o una ambientación de buenos deseos. Más allá, esta ha de proveer a sus alumnos la integridad y calidad moral, el descubrimiento de su vida sobrenatural, y el servicio hacia el otro, como obras santificadoras (Argandoña 2011). Es así que, la universidad adquiere un valor vital si facilita este encuentro, con quien da respuesta a los interrogantes más existenciales del ser humano. Y esto, pasa ineludiblemente a través de favorecer la participación en la esencia misma de Dios con la Verdad revelada en Jesucristo, piedra angular de los partícipes de los bienes gratuitos, tanto naturales como la inteligencia, libertad, y la voluntad; preternaturales como la inmortalidad; y sobrenaturales como la gracia y la gloria.

Que éste les haga sentir y vivir como piezas fundamentales de la obra de Dios, garante de su verdadero desarrollo, en una existencia virtuosa, de renovada conciencia, y de su experiencia interiorizada como cristianos. Llamados a una conversión interior, y desde las virtudes y la experiencia espiritual de veinte siglos, han de contemplar agradecidos la fragilidad propia y de su entorno, con una visión respetuosa y crítica, que les haga brotar la reacción moral y la autotrascendencia, rompiendo su conciencia aislada. Que sea desde las ciencias que atienden a la salud del cuerpo, a la salud social, o a la espiritual, esto es, desde todas aquellas Facultades académicas que han sido la cuna de nuestra civilización en sus albores, de donde brote una conciencia de 'casa común', universal, de mutua pertenencia y futuro compartido. Ya el Papa Francisco nos proveyó de una lección magistral en su Carta Encíclica Laudato si', donde en atención a los valores humanos, afirmaba que: "no basta hablar sólo de la integridad de los ecosistemas. Hay 
que atreverse a hablar de la integridad de la vida humana, de la necesidad de alentar y conjugar todos los grandes valores" (Francisco 2015, 225). Y cuando se estudia la conducta humana de forma sistemática, examinada a la luz de los valores y principios morales, estamos hablando de Bioética (Reich 1995); esto es: de la ética aplicada a la vida humana.

Así como la docencia y la investigación son funciones íntimamente ligadas a la universidad, la bioética y la sostenibilidad van de la mano (Contreras-Islas et al. 2016). La universidad como ente aglutinador de estos valores, debe también ser facilitadora del paradigma relacional ser humano-sociedad-medioambiente, aunque en la actualidad se encuentra sumida, en contraposición, en la indefinición del constructo sostenible, dando lugar a un desinterés o desinformación por parte del claustro académico; o por el contrario, a una ingente diversidad de propuestas ante su interpretación ad hoc del término "emprendimiento sostenible", su confesionalidad o no. Estos perfiles establecen sus prioridades para el logro del equilibrio entre los sistemas sociales, culturales y económicos. El corolario laicista más actual, es un discurso marcado con valores mayoritariamente ecocéntricos o antropocéntricos, de corte financiero y tecnocrático, reforzado con una serie de marketing e imagen, conducentes a una reductio ad absurdum que permite, tal como expresa en sus conclusiones David Alba: "A una laxitud impropia de la relevancia del reto, que puede desencadenar malas prácticas, contrarias incluso a los principios que defienden” (Alba 2015, 217).

\section{La Revelación Cristiana como germen ético-sostenible}

La Verdad revelada por los textos sagrados es tan inspiradora y sugerente como el axioma de que las cuestiones éticas ni prescinden, ni pueden responderse siempre desde el conocimiento científico. El sujeto no puede reducirse a la categoría de objeto, por lo que resulta necesaria la interdisciplinariedad para la mayor comprensión del bien común. Vittorio Hösle expresa con radicalidad, que: "la ética trasciende la ciencia; y solo cuando esta, de modo erróneo, se toma a sí misma como la totalidad de la racionalidad humana, puede llegar a la convicción de que las proposiciones éticas no solo no son 
científicas, sino tampoco racionales" (Hösle 2018, 16). Karol Wojtyla enunciaba en su Tesis de Habilitación, que: "Según el Evangelio, hemos de buscar el contenido ético de la vida en la actividad que se produce en lo interior, en lo profundo de la persona, mediante el amor" (Wojtyla 1982, 5). Resulta por tanto ineludible este principio para la correcta interpretación del bien y el mal, y para el perfeccionamiento moral; antítesis del antropocentrismo despótico, al proponer la necesaria relación con Dios, con el prójimo y con la Tierra, trascendentales para la existencia. Ante el postulado según Berry de que "la preocupación por el Cristo histórico no es en este momento servicial” (Berry 1991, 75), urge anunciar el pensamiento tomista de que son las palabras de Cristo las que preservan la vida integral en plenitud (George 2019). Y esta es la justificación y pertinencia de implementar al servicio de la comunidad académica, la denominada Educación Integral, como materia normativa, en íntima relación con una antropología cristiana, capaz de favorecer el capital ético en valores morales, el bienestar personal, social, y del ecosistema, con una profunda consciencia autónoma y responsable. De nada nos sirven fórmulas seudocientíficas, de amables y oportunistas newagers. El relativismo práctico nos ha llevado más que nunca a tal consideración (Francisco 2015, 122). Cuando el hombre o la mujer, "eternos aspirantes" a ser dios, creen ser el centro de todo, el resto es circunstancial y relativo. Más aún si se conducen inmersos en una antítesis al monoteísmo trinitario, como el concepto pancosmista búdico de la divinidad que despersonaliza al Cristo histórico, que iguala al ser humano con los animales, las plantas o las cosas, y que reduce todo a una conciencia energética universal. Este pensamiento, propio de movimientos como Nueva Era, es concebido como un "autismo espiritual", al que en palabras del Papa Francisco en su homilía del 20 de julio de 2014: "ni Jesús hubiera creído". Al contrario, si ese ser humano tiene a Cristo como referente, intérprete de la vida, de la Creación, y del sentido de su ser, éste es "guiado" por el Paráclito desde ese perfil Cristocéntrico al que es configurado (Pineda-Albaladejo et al. 2016), y donde la vida es un don, que conlleva de forma inherente, una conciencia ética, un bienestar, un compromiso y una responsabilidad moral, de intenciones y conductas éticamente sostenibles (Juan Pablo II 1979; Pablo VI 1965). 
Perfil Cristocéntrico, como germen fruto de la fe que, de forma inherente, identifica e internaliza la dimensión ontológica, esto es, la naturaleza del ser humano como ser vivo, epistemológica como proceso racional e intelectivo, y axiológica, como reconocimiento de los valores que encumbran su dignidad. Estas dimensiones sustentan y orientan hacia una práctica reflexionada que de sentido a la acción: la dimensión praxeológica (Bedart 2003), atenuándose así la carga técnica-utilitaria heredada de la modernidad, a través de la capacitación en aptitudes de razonamiento, de investigación y conceptualización, de formulación y de dispositivos, como formas de reflexión crítica.

Añadía Karold Wojtyla que: "los valores, al plasmar la experiencia desde su mismo interior, influyen sobre su decurso, es decir, disponen todos los elementos reales de la experiencia de un modo peculiar” (Wojtyla 1982, 8). Resulta, por tanto, que si nos centráramos en la experiencia humana sin tener en cuenta el momento axiológico-normativo, nos situaríamos o en la introspección propia de la Psicología, o en la Fenomenología como método de inferir la constante ética tal decurso de los valores éticos manifestados en las experiencias. Esto queda a medio camino del análisis de la experiencia ética humana, al no quedar definido el principio objetivo por el que un acto de la persona es éticamente bueno o no. Es por ello, que el definir el bien o el mal a la luz de dicho principio, nos conduciría al método metafísico: al orden cristiano revelado. Más allá de la definición del valor ético manifestado en la conducta, se nos hace necesaria la búsqueda de dicho valor del orden moral objetivo, a la perfección suprema, al que la ética cristiana apela como su principio objetivo, tal como apunta Jacques Maritain: “No hay una fundación primaria e inamovible del carácter incondicional de la ley moral y de la obligación moral, excepto Dios” (Maritain 1958, 2).

\section{El valor de la dimensión espiritual}

¿La espiritualidad que emana de la propia Educación Integral es un factor sinérgico de bienestar y valores sostenibles? Los estudios más recientes de la literatura científica nos invitan, no solo a tener en cuenta diferentes factores de orden tanto biológico, psicológico, o social, sino principalmente 
al orden espiritual, a la hora de investigar los valores y actitudes que median la intención, y que dan como experiencia tangible, la conducta ética o deontológica en cada contexto. Cita Soares en las conclusiones generales de su investigación que: "No hay bienestar físico sin bienestar psicológico, ni bienestar psicológico sin bienestar físico, ni ambos sin un bienestar espiritual" (Soares 2015, 173) Resulta obvio deducir que, a pesar de la dicotomía entre el deseo y el acto final, a la que se enfrenta el ser humano como constante desafío, existe una probabilidad mayor de que una recta conciencia de como fruto una ordenada intención, que un acto moralmente ilícito o considerado malo. Desde este razonamiento inductivo-transitivo podemos teorizar que, un bienestar espiritual y psicológico conforma un constructo con mayor previsión de buena salud física y social, por ende, un mejor capital ético en valores que puedan ser compartidos de forma sostenible.

Apoyándonos en las dimensiones del citado constructo "Perfil Cristocéntrico", verificamos el valor de la propia espiritualidad, evidenciando la imbricación y la interdependencia de los componentes implicados, donde sus factores se asocian de forma positiva y significativa. El Bienestar Espiritual es investigado desde la propia importancia de las creencias y sus prácticas, como promotoras de sabiduría y entendimiento, desde la experiencia personal del ser, el saber y el hacer. La necesidad espiritual, como fuerza unificante de las dimensiones físicas, emocionales y sociales en su relación con la alteridad, da como fruto una autoconciencia que da luz a la visión de uno mismo.

Espiritualidad incubadora de Bienestar Psicológico, donde la autoaceptación resulta uno de los criterios centrales, que aúna actitudes positivas hacia sí mismo, seguridad, confianza y valoración de su propia historia. Que favorece las relaciones positivas, estrechas y cálidas, con empatía e intimidad, donde el otro pasa a ser una cuestión fundamental. Que alienta la autodeterminación de las conductas, su independencia, y autorregulación del comportamiento, como conceptos que identifican a la autonomía. Que asiste al dominio del entorno de forma electa y creativa, beneficiando el aprovechamiento de oportunidades. Esto da lugar a un mayor potencial de autoconocimiento, de actitudes y capacidades, con su dote de sentido 
existencial. En suma, se verifica una mayor aportación al crecimiento personal y al propósito en la vida.

En relación con las Actitudes de Emprendimiento, genera un mayor nivel de actitud creativa y generadora de iniciativas, con mejoras y asunción de responsabilidad y control, propios de la proactividad. Mayor capacidad de creación, selección, organización, y uso de los recursos, que identifican a la innovación, con un alto nivel ético profesional, empático, y con asunción de riesgos de forma responsable.

Si la espiritualidad resulta relevante para el propio bienestar espiritual, psicológico y al emprendimiento, lo es también para las Actitudes Sostenibles y para el comportamiento pro-ecológico (Csutora and Zsóka 2012; Félix and Braunsberger 2016). La verdad sostenible, como visión integral del hombre y del ecosistema, educada y cultivada en solidas virtudes, implica la voluntad de cambio y el crecimiento interior de cada individuo (Hedlund-de Witt, De Boer and Boersema 2014), con la necesaria trascendencia, y en estrecha relación con factores propios de la espiritualidad de la Iglesia, como la frugalidad, el comportamiento pro-social y pro-ecológico, el altruismo y la equidad, entre otros (Barrera 2017).

Otros factores como la percepción de normas ambientales, la deliberación, afinidad, austeridad, altruismo, o la conducta ecológica general, son favorecidas por la espiritualidad de forma sinérgica. Es por ello que, ante las propuestas actuales de cambios en estructuras sociales de los sistemas de producción, más propias de paradigmas medio-ambientalistas, nos urgen nuevos planteamientos que armonicen la relación de las conductas humanas con la sociedad y con la naturaleza, desde una ética integral. El supuesto sostenible nos plantea una evolución de la relación del ser humano, y el sistema natural con el que convive. Relación con fundamentos éticos, basados en principios morales como la justicia, responsabilidad, equidad, y solidaridad humana y ecológica. Para ello se ha de promover una nueva conciencia bioética y existencial, que contemple lo ontológico del ser humano, su inherente riqueza espiritual, como umbral de las intenciones y actos inspirados de Dios, creador de todo, donde la Educación Integral contiene suficientes argumentos y fundamentos para ello. 


\section{Conclusiones}

Hemos expuesto que la función que desempeña el ente universitario, en cuanto al enriquecimiento del capital humano, tanto en sus dimensiones biológica, psicológica, social y espiritual del alumno, resulta vital, y un factor clave donde la comunidad científica debe examinar el impacto en la práctica, sobre los futuros profesionales, y no tanto la veracidad o consistencia de las creencias que implícitamente se imparten en las materias de la denominada Educación Integral.

La Educación Integral constituye un factor potencial, que no infalible, contribuyente al bienestar psicológico y espiritual, así como a un cambio en favor de actitudes y conductas emprendedoras y sostenibles en los sujetos, desde una visión armónica, impregnada del justo orden de valores éticos y morales. Ello nos aproxima a la configuración del perfil denominado "Cristocéntrico", como actitud representativa de una sólida base moral, e intereses enfocados hacia el ser humano, el bien colectivo, el respeto hacia el ecosistema, y hacia la propia religiosidad de forma prioritaria, como sugiere dicha formación.

Ante la urgente necesidad de una nueva visión y modelo de enseñanza superior, procede la incorporación en todos los currículos académicos, materias promotoras de actitudes y competencias sobre emprendimiento sostenible de forma integral, con los indicadores establecidos por los organismos internacionales en dicha materia. La formación del profesorado resulta esencial ya que éstos, además de ayudar al desarrollo cognitivo, psicomotriz y actitudinal de sus alumnos, deben facilitar el diálogo entre ciencia y fe a través de la razón, como referente común; en síntesis: saber juzgar, evaluar, emprender, y actuar, con la exigencia de una reflexión ética-moral en sus distintas disciplinas, como razón trascendente de su enseñanza.

\section{Referencias}

Alba, David. 2015. "La evaluación de la contribución de la universidad a la sostenibilidad ambiental. Una aplicación a las universidades españolas”. Tesis Doctoral, Universidad Autónoma de Madrid. 
Argandoña, Antonio. 2011. “Josemaría Escrivá de Balaguer y la misión del IESE en el mundo de la empresa”. Studia et Documenta: Rivista dell'Istituto Storico San Josemaría Escrivá, 5: 131-164. https://dialnet.unirioja.es/servlet/articulo?codigo=3636036 [Consulta: 29/03/2019].

Barrera, Laura Fernanda. 2017. “Espiritualidad y Elementos Físicos que la Propician, Conductas Sustentables, Conservación Ambiental y Bienestar: Un Modelo de Ambiente Espiritual Positivo”. Tesis Doctoral, Universidad de Sonora. México. Bédard, Renée. 2003. “Los fundamentos del pensamiento y las prácticas administrativas: el rombo y las cuatro dimensiones filosóficas”. Revista Ad-Minister, 3: 68-88. http://publicaciones.eafit.edu.co/index.php/administer/article/view/697/621 [Consulta: 29/03/2019].

Benedicto XVI. 2009. “Carta encíclica Caritas in veritate, sobre el desarrollo humano integral en la caridad y en la verdad”. Roma. http://www.vatican.va/holy_father/ benedict_xvi/encyclicals/documents/hf_ben-xvi_enc_20090629_caritas-in-veritate_sp.html [Consulta: 29/03/2019].

Berry, Thomas, and Thomas Clarke. 1991. "Befriending the Earth: A Theology of Reconciliation Between Humans and the Earth". London: Twenty-Third Publications.

Congregación para la Doctrina de la Fe. 2008. “Instr. Dignitas personae sobre algunas cuestiones de bioética”. Roma, 100: 858-887. http://www.vatican.va/roman_curia/ congregations/cfaith/documents/rc_con_cfaith_doc_20081212_sintesi-dignitas-personae_sp.html [Consulta: 29/03/2019].

Contreras-Islas, David Sebastián et al. 2016. “Diagnóstico de componentes bioéticos en el ámbito educativo de Ciencias Biológicas y de la Salud”. Revista Latinoamericana de Bioética, 17(1): 14-35. DOI: 10.18359/rlbi.2137.

Csutora, María, and Ágnes Zsóka. 2012. "Relation of spirituality to happiness, life satisfaction and sustainable lifestyles”. En Annals of the International Conference on Spirituality and Sustainability: A New Path for Entrepreneurship. Visegrád, Hungría. https://core.ac.uk/download/files/481/12355453.pdf [Consulta: 29/03/2019].

Felix, Reto, and Karin Braunsberger. 2016. "I believe therefore I care: the relationship between religiosity, environmental attitudes, and green product purchase in Mexico”. International Marketing Review, 33(1):137-155. DOI: 10.1108/IMR07-2014-0216.

Francisco. 2015. “Carta Encíclica Laudato si’”. Roma. http://w2.vatican.va/content/ francesco/es/encyclicals/documents/papa-francesco_20150524_enciclica-laudato-si.html [Consulta: 29/03/19].

George, Marie. 2019. “Is Eco-Theologian Thomas Berry a Thomist? Scientia et Fides, 7(1), 47-71. DOI: $10.12775 /$ SetF.2019.003 
Hedlund-de Witt, Annik, Joop De Boer, and Jan J. Boersema. 2014. “Exploring inner and outer worlds: A quantitative study of worldviews, environmental attitudes, and sustainable lifestyles”. Journal of Environmental Psychology, 37: 40-54. DOI: 10.1016/j.jenvp.2013.11.005.

Hösle, Vittorio. 2018. “La Idea de Universidad ante los Desafíos del Siglo XXI”. Documentos Core Curriculum. Universidad de Navarra. http://hdl.handle. net/10171/52822 [Consulta: 29/03/2019].

Juan Pablo II. 1979. “Carta Encíclica Redemptoris Hominis”. Roma. http://w2.vatican. va/content/john-paul-ii/es/encyclicals/documents/hf_jp-ii_enc_04031979_redemptor-hominis.html [Consulta: 29/03/2019].

Lipovetsky, Gilles. 2007. “La felicidad paradójica. Ensayo sobre la sociedad del hiperconsumo”. Barcelona: Anagrama.

Lozano, Rodrigo et al. 2015. "A review of commitment and implementation of sustainable development in higher education: results from a worldwide survey". Journal of Cleaner Production, 108: 1-18. DOI: 10.1016/j.jclepro.2014.09.048.

Maritain, Jacques. 1943. “Una educación Integral para un Humanismo Integral”. 1943. Obras Breves de Jacques Maritain, 040-04. http://www.jacquesmaritain. com/pdf/10_EDU/04_ED_EduHI.pdf [Consulta: 29/03/2019].

Maritain, Jean. 1958. “Aspectos Morales y Espirituales de la Educación”. Obras Breves de Jacques Maritain, 040-A1. http://www.jacquesmaritain.com/pdf/10_EDU/06_ ED_EduMoral.pdf. [Consulta: 29/03/2019].

Martínez, Javier. 2019. “La Teología de la Creación a la Luz de la Ciencia. Presente y Futuro en la Constante Tarea de Renovar la Teología de la Creación”. Scientia et Fides, 7(1), 183-205. DOI: 10.12775/SetF. 2019.009

Pablo VI. 1965. “Carta Encíclica Gaudium et Spes”. Roma. http://www.vatican.va/ archive/hist_councils/ii_vatican_council/documents/vat-ii_const_19651207_gaudium-et-spes_sp.html [Consulta: 29/03/2019].

Pineda-Albaladejo, Juan Manuel, Francisco J. Moya-Faz, and Jorge López. 2017. “Training in Values as an Incubator for Sustainability Attitudes”. Harvard Deusto Business Research, 6(2): 60-67. DOI: 10.3926/hdbr.84.

Pineda-Albaladejo, Juan Manuel, and Alberto Sanz. 2016. "Christcentric Profile vs New Age: the Transgression of the Sustainable Truth”. Comunicación presentada al Congreso Internacional Laudato Si'. UCAM.

Puig, Jordi. 2019. "Sensibilidad por el medio ambiente y cristianismo". Scientia et Fides, 7(1), 73-96. DOI: 10.12775/SetF. 2019.004

Reich, Warren T. (Ed.). 1995. “Encyclopedia of Bioethics”. Nueva York: Simon \& Schuster MacMillan. 
Saad, Gad. 2007. “The evolutionary bases of consumption”. Mahwah, NY: Lawrence Erlbaum Associates.

Serra, Miguel A. 2012. "Ética, Bioética y el Educador en la Enseñanza de las Ciencias Clínicas”. Educación Médica Superior, 26(3): 425-433.

Soares, María M. 2015. “Influencia de las Creencias Personales en el Bienestar Físico, Psicológico y Emocional desde una Perspectiva General”. Tesis Doctoral, Universidad de León. https://buleria.unileon.es/bitstream/handle/10612/5892/ Tesis\%20Manuela\%20Soares.pdf?sequence=1 [Consulta: 29/03/2019].

UNESCO. 2007. “Decenio de las Naciones Unidas de la Educación para el Desarrollo Sostenible 2005-2014. El Decenio en pocas palabras”. París: UNESCO. http:// unesdoc.unesco.org/images/0014/001416/141629s.pdf [Consulta: 29/03/2019]. Wojtyla, Karol. 1982. “Max Scheler y la Ética Cristiana”. Madrid: Biblioteca de Autores Cristianos. http://www.jacquesmaritain.com/pdf/23_OA/3T-4.1.pdf [Consulta: 29/03/2019]. 